\title{
Caracterización productiva y organoléptica de huevos de gallinas de campo de la región sierra del Ecuador
}

\author{
Toalombo, P.A. ${ }^{1,2 @ ; ~ N a v a s-G o n z a ́ l e z, ~ F . J . ~}{ }^{2}$; Andrade-Yucailla, V.C. ${ }^{3}$; Trujillo, J.V. ${ }^{1}$; Martinez, J. ${ }^{1}$ y Delgado, J.V. ${ }^{2}$
}

'Escuela Superior de Chimborazo, Carrera de Zootecnia, Riobamba. Ecuador.

${ }^{2}$ Departamento de Genética. Universidad de Córdoba. España.

${ }^{3}$ Universidad Estatal Amazónica, Centro de Investigación Posgrado y Conservación Amazónica, Pastaza. Ecuador.

\section{Palabras CLAVE ADICIONALES}

Gallinas de campo.

Saberes ancestrales.

Recursos genéticos locales.

Características fanerópticas.

Producción sustentable.

\section{RESUMEN}

En la Estación Experimental Tunshi de la Escuela Superior Politécnica de Chimborazo, parroquia Licto, a $2712 \mathrm{msnm}$ a una temperatura promedio de $14,92^{\circ} \mathrm{C}$. Se evaluó el comportamiento productivo para huevos de gallinas de campo y se analizó las características organolépticas, en 94 aves de traspatios identificadas en la región sierra del Ecuador de las provincias: Pichincha, Bolívar, Loja, Cañar, Tungurahua, y Chimborazo, una vez adaptadas in situ, la investigación tuvo una duración de 120 días. Los datos recogidos fueron tabulados en el programa estadístico SPSS versión Statistic 19, se obtuvo estadísticos descriptivos. Los resultados para peso inicial promedio fue de 1508,78 g; peso final 1502,36 $\pm 247,61$ g; ganancia de peso 175,26 $\pm 137,20 \mathrm{~g}$; Los parámetros de calidad del huevo fueron: peso promedio $54,95 \pm 5,24 \mathrm{~g}$; masa del huevo de 48,15 $\pm 4,68 \mathrm{~g}$; ancho del huevo de 42,25 mm; longitud del huevo de 55,40 $\pm 2,60 \mathrm{~mm}$; peso de la cáscara de 6,80 g; grosor de la cáscara de 0,32 $\pm 0,02 \mathrm{~mm}$. Para la medición organoléptica se realizó un análisis sensorial triangular, utilizando 6 combinaciones con 3 repeticiones, total 18 catadores, estos resultados fueron sometidos a Chi-cuadrado obteniendo diferencias significativas en sabor del huevo de campo con relación al comercial. Cabe indicar que la característica de rusticidad en las gallinas, representa un factor importante para la conservación de recursos genéticos, ya que han demostrado poseer una capacidad de adaptación al medio ambiente, que aseguran buenos rendimientos productivos y que resultan interesantes para la seguridad y soberanía alimentaria.

\section{Productive and organoleptic characterization of eggs from field hens in the sierra region of Ecuador}

\section{SUMMARY}

\section{ADDITIONAL KEYWORDS}

Creole chickens.

Ancestral knowledge.

Local genetic resources.

Phaneroptics characteristics.

Sustainable production.

\section{INFORMATION}

Cronología del artíiulo.

Recibido/Received: 03.02.2019

Aceptado/Accepted: 08.09.2019

On-line: 15.07.2019

Correspondencia a los autores/Contact e-mail:

ptoalombo@espoch.edu.ec
At the Tunshi Experimental Station of the Escuela Superior Politécnica de Chimborazo, Licto, at 2712 meters above sea level at an average temperature of $14.92{ }^{\circ} \mathrm{C}$. The productive behavior for eggs of field hens was evaluated and the organoleptic characteristics were analyzed, in 94 birds of backyards identified in the mountain region of Ecuador of the provinces: Pichincha, Bolívar, Loja, Cañar, Tungurahua, and Chimborazo, once adapted In situ, the investigation lasted 120 days. The data collected were tabulated in the statistical program SPSS Statistic version 19, descriptive statistics were obtained. The results for average initial weight was $1508.78 \mathrm{~g}$; final weight $1502.36 \pm 247.61 \mathrm{~g}$; weight gain $175.26 \pm 137.20 \mathrm{~g}$; The parameters of egg quality were: average weight $54.95 \pm 5.24 \mathrm{~g}$; egg mass of $48.15 \pm 4.68 \mathrm{~g}$; egg width $42.25 \mathrm{~mm}$; egg length of $55.40 \pm 2.60 \mathrm{~mm}$; shell weight of $6.80 \mathrm{~g}$; thickness of the shell of $0.32 \pm 0.02 \mathrm{~mm}$. For the organoleptic measurement, a triangular sensory analysis was carried out, using 6 combinations with 3 repetitions, total 18 tasters, these results were subjected to Chi-square, obtaining significant differences in the flavor of the field egg in relation to the commercial one. It should be noted that the characteristic of hardiness in chickens represents an important factor for the conservation of genetic resources, since they have shown to have a capacity to adapt to the environment, which ensure good productive yields and which are interesting for food security and sovereignty.

\section{INTRODUCCIÓN}

La avicultura rural es una actividad de importancia social, cultural y económica, por constituirse en una fuente de alimento para las familias campesinas que ayuda a complementar los requerimientos nutricio- nales, además es un importante recurso zoogenético del país (Alderson 2018), (Andrade-Yucailla V. \& Toalombo-Vargas P. 2018), (Villacís Rivas et al. 2016); la cría de animales menores se convierte en la caja menor campesina (Rodríguez García 2007), ya que aporta a los 
recursos familiares. Según, (FAO 2015) la producción mundial de huevos en el año 1983 fue de 29,3 millones de toneladas, considerándose un logro para la década de los 80, sin embargo, hasta la actualidad esta producción ha superado en 44,5 millones de toneladas destacándose los países de América Central y Este de Asia como los mayores productores de dicho alimento, de la misma manera señala que el huevo tiene un alto valor nutritivo al aportar proteína, energía, vitaminas y minerales, siendo uno de los alimentos de origen animal completos; y por poseer dichas características nutritivas y al ser de bajo costo, éste alimento ha sido difundido en una multitud de civilizaciones y culturas. En base a (FAO 2015), indica que entre los años 2000 a 2016 la producción mundial de huevos se ha incrementado en un $42 \%$, a una tasa promedio anual de 2,2\%, en 2016 alcanzó 1.360 billones de unidades de huevos, un $1,3 \%$ más que en 2015, que corresponde a la especie (Gallus domesticus) y de manera alternativa se consumen en menor cantidad huevos de otras especies como codornices, pavo, patos y ganzos. (Primo-Yúfera, 1997), los huevos camperos son criados a campo abierto de manera libre, con restos de alimentos orgánicosecológicos, a partir de granos, pastos de la zona y demás recursos que se producen en una parcela, que se encuentran a disposición del lugar (Rodríguez García 2007); además el sistema de producción implementado brinda un estado de bienestar animal, que influye de manera positiva en los procesos metabólicos y por ende en la producción. Sin embargo, este tipo de explotación siendo tradicional con un manejo técnico mínimo y sin los adecuados planes de desparasitación, se ven reflejados en una baja producción y muerte de los animales, limitando su productividad (Luka \& Ndams 2007).

Ecuador al estar ubicado en la zona de la cual lleva su nombre (ecuatorial) y al mismo tiempo estar atravesado por la cordillera de los Andes, presenta diferentes zonas agroecológicas y diferentes pisos climáticos que lo hacen rico en biodiversidad, tanto de especies exóticas como domésticas; es así el caso de las gallinas de campo, de las cuales no existe un banco de información divulgada; aun cuando son la base genética de fácil adaptabilidad, rusticidad y que en la actualidad están en peligro de desaparecer (Moula et al. 2014), constituyen la base de la seguridad y soberanía alimentaria, por lo que se justifica la necesidad de investigar sobre el comportamiento productivo y caracterización organoléptica de huevos de gallinas de campo presentes en traspatios de la región sierra del Ecuador con miras a la creación de núcleos genéticos y bancos de germoplasma (Formoso-Rafferty et al. 2016).

\section{MATERIAL Y MÉTODOS}

La presente investigación fue realizada en la Estación Experimental Tunshi de la Escuela Superior Politécnica de Chimborazo, ubicada en la parroquia Lícto, a $1^{\circ} 44^{\prime} 54.1^{\prime \prime} \mathrm{S} 78^{\circ} 37^{\prime} 32.6^{\prime \prime} \mathrm{W}, 2712 \mathrm{msnm}$ a una temperatura de $14,92{ }^{\circ} \mathrm{C}$ aproximadamente; para el levantamiento de información se identificó 94 aves de campo de las provincias de Pichincha, Bolívar, Loja, Cañar, Tungurahua y Chimborazo; una vez adaptadas in situ, la duración del experimento fue de 120 días. Las aves fueron manejadas bajo un sistema extensivo, alimentadas con pienso + residuos de cosecha + pastoreo y agua ad libitum. Las variables estudiadas fueron sometidas a un análisis estadístico descriptivo siendo: Peso del Huevo (PH), Masa del huevo (MH), Peso de la cascara (PC), Grosor de la cascara (GC), Ancho del huevo $(\mathrm{AH})$ y Largo del huevo (LH), con una balanza y pie de rey digital. Para las variables organolépticas, se realizó un análisis sensorial triangular, utilizando 6 combinaciones con 3 repeticiones, total 18 catadores, los datos fueron sometidos a Chi-cuadrado; analizados con el programa estadístico SPSS versión Statistic 18.

\section{RESULTADOS}

Los resultados obtenidos en el estudio sobre el comportamiento productivo de las gallinas criollas de la región Sierra de Ecuador en el peso inicial se presentaron los siguientes resultados por provincia: Bolívar un promedio de 1612,00 $\pm 442,15 \mathrm{~g}$; Cañar 1357,5 \pm 374,36 g; Chimborazo 1280,56 $\pm 327,10 \mathrm{~g}$; Loja 1864,76 $\pm 795,37$ g; Pichincha 1467,86 \pm 484,22 g; Tungurahua $1470,00 \pm 327,62 \mathrm{~g}$.

Las ganancias de peso de las gallinas de campo son reportadas para verificar el comportamiento productivo de las aves, las que fueron tomadas en su madurez sexual, por tal motivo no se espera una notable ganancia de peso en sus respectivas provincias: Bolívar registra una de ganancia de $257 \pm 95,68 \mathrm{~g}$; en Cañar $289 \pm 142,14$; Chimborazo 76,67 \pm 92,91 g; Loja 252,50

Tabla I. Porcentaje de producción de huevos de gallinas criollas presentes en la región sierra del Ecuador (Percentage of egg production of Creole hens present in the region Mountain range of Ecuador).

\begin{tabular}{lcccc}
\hline Provincia & $\mathrm{N}^{\circ}$ gallinas & $\mathrm{N}^{\circ}$ de huevos & Producción (\%) & Huevos/gallina \\
\hline Pichincha & 12 & 309 & 13,5 & 25,75 \\
Bolivar & 14 & 488 & 21,4 & 34,86 \\
Loja & 21 & 513 & 22,5 & 24,43 \\
Cañar & 17 & 476 & 20,8 & 28 \\
Tungurahua & 9 & 269 & 11,8 & 29,89 \\
Chimborazo & 8 & 228 & 10 & 28,5 \\
TOTAL & 81 & 2283 & 16,7 & \\
\hline
\end{tabular}

Media: 28,57

DS: 3,65 
\pm 209,49 g; Pichincha 205,40 \pm 118,33 g; Tungurahua $118,00 \pm 96,98 \mathrm{~g}$; al finalizar la investigación en las gallinas de campo de la Región Sierra, se alcanzaron los siguientes pesos finales por provincia: Bolívar un promedio de 1625,36 \pm 498,40 g; Cañar 1419,94 \pm 375,28 g; Chimborazo 1170,88 \pm 277,77 g; Loja 1909,90 \pm 824,33 g; Pichincha 1474,42 \pm 442,39 g; Tungurahua 1413,67 \pm $276,01 \mathrm{~g}$, con un promedio total de 1502,36 $\pm 247,6 \mathrm{~g}$.

En lo que respecta a la producción de huevos el total de producción fue de $16,67 \%$, equivalente a 2283 huevos, el número de huevos/gallina fue de 28,57 $\pm 3,65$ conforme el periodo de estudio que duró la investigación, como se puede observar en Tabla I. Es importante mencionar el estrés por adaptación al ser trasladadas al lugar de investigación, influyó en la producción de huevos. Al analizar el porcentaje de producción, se obtuvo los siguientes resultados en $\%$ de huevos: Loja 22,50; Bolívar 21,40; Cañar 20,80; Pichincha 13,50; Tungurahua 11,80 y Chimborazo 10,00.

En lo referente al peso del huevo $(\mathrm{g})$, se determinó un promedio de 54,95 \pm 5,24 g Tabla II, resultados fundamentados en una alimentación de balanceado + residuos de cosecha + pastoreo. Para masa del huevo (g) de las gallinas criollas presentes en la Sierra ecuatoriana, se obtuvo un promedio de 48,15 $\pm 4,68 \mathrm{~g}$. Al analizar la variable ancho del huevo de las gallinas criollas de la Sierra ecuatoriana, se obtuvo un promedio de $42,45 \pm 1,36 \mathrm{~g}$.

En la evaluación organoléptica, se determinó a través de encuestas, utilizando el método triangular para el sabor del huevo, comparando el sabor del huevo de gallina criolla con el huevo de gallina comercial, para el cual se realizaron 6 posibles combinaciones con 3 repeticiones, en total 18 catadores fueron sometidos a la degustación, obteniéndose los siguientes resultados: aciertos 8 y desaciertos 10 . Al someter los resultados a la prueba de Chi cuadrado se obtuvieron diferencias significativas, entre el sabor del huevo de gallina de campo y el huevo de gallina comercial Tabla III. La Hipótesis se acepta: Chi cuadrado calculado es menor que Chi cuadrado tabular, por lo tanto, existe diferencia entre el sabor del huevo criollo y el huevo de gallina comercial.

\section{DISCUSIÓN}

Los pesos iniciales son similares a los reportados por (Salas et al. 2009), pesos entre 1400 a 1600 g, esto

Tabla II. Parámetros de calidad de huevo de las gallinas criollas presentes en la región sierra del Ecuador (Parameters of egg quality of the Creole hens present in the region Mountain range of Ecuador.)

\begin{tabular}{lcc}
\hline Parámetros & Media & DS \\
\hline Peso del huevo, $(\mathrm{g})$ & 54,95 & 5,24 \\
Masa del huevo, $(\mathrm{g})$ & 48,15 & 4,68 \\
Peso de la cáscara, $(\mathrm{g})$ & 6,80 & 1,11 \\
Grosor de la cáscara, (mm) & 0,32 & 0,02 \\
Ancho del huevo, (mm) & 42,45 & 1,36 \\
Largo del huevo, $(\mathrm{mm})$ & 55,4 & 2,60 \\
\hline
\end{tabular}

se debe a que el ensayo se realizó en una zona análoga, entre 2000 y 3000 msnm; similares forma reporta (Vignon \& Santiago 1996), en un sistema de traspatio, en donde las gallinas criollas de los diferentes grupos evaluados tuvieron un peso promedio de $1300 \mathrm{~g}$ esto se debe al tipo de alimentación utilizado a base de maíz y además lo disponible en el piso como larvas de insectos, desperdicios de cocina, desechos de cosecha, entre otros y (Lázaro et al. 2012), reporta un peso promedio de 1603,30 $\pm 3,20 \mathrm{~g}$, este valor es mayor a lo reportado en la presente investigación, lo que indica una adaptación de las diferentes cruzas de aves que manejan los productores en clima templado. Los resultados obtenidos difieren a los reportados por (Toscano 2002), quien menciona que las gallinas criollas alimentadas con una dieta a base de maíz, tuvieron un peso vivo de 2044,00 g; y las alimentadas con trigo pesaron 2016,00 g, a las 35 semanas de edad evaluadas bajo un sistema semiintensivo en su lugar de origen, claramente indica que las diferencias encontradas se deben a la adaptación, alimentación y sistema de producción aplicado, en esta investigación, que fue bajo un sistema extensivo, dieta a base de balanceado + residuos de cosecha + pastoreo a voluntad, durante el periodo de experimentación; las aves se encontraban en un ambiente libre y el gasto de energía fue elevado.

En cuanto a la producción de huevos estos valores son similares a los reportados por (Salas et al.), en la producción de 40 gallinas criollas donde obtuvo un total acumulado de 903 huevos durante 19 semanas que duró su investigación, lo que significa una producción de 22,5 huevos/ave (Rodríguez Buenfil et al. 1996); valores inferiores a los obtenidos en la presente investigación y difiere con lo mencionado por (Rodríguez Buenfil et al. 1996) (Andrade-Yucailla V. \& ToalomboVargas P. 2018) que reporta una producción entre 35 y 47 huevos por gallina en un periodo de 24 semanas, valores mayores a los obtenidos en esta investigación.

En cuanto al peso de los huevos este valor son similares a los reportados a los de (Andrade-Yucailla V. \& Toalombo-Vargas P. 2018) y superior a los reportado en el peso promedio del huevo a la postura, 53,30 g, obtenido por (Vignon 1997), en un trabajo con gallinas criollas bajo un sistema semi-intensivo; así mismo, un valor mayor a lo reportado por (Monterrubio 2000), quién evalúo gallinas criollas bajo una dieta de maíz, el peso del huevo fue de 51,90 g; pero los resultados son inferiores a los obtenidos por (Martínez Correal 2010), con dieta de maíz y trigo donde obtuvo pesos de huevo a la postura de 55,30 y 54,60 g respectivamente y (Toscano 2002), obtuvo pesos de huevo a la postura con dieta de maíz y trigo de 59,10 y 55,10 g, correspondientemente.

Para masa de huevo los resultados obtenidos son superiores a los reportados en gallinas criollas criadas en traspatio en Michoacán - México (Juárez-Caratachea et al. 2010), donde registró una masa inferior del huevo con un promedio de 46,20 g. Al estudiar la variable peso de la cáscara en las gallinas criollas de la Sierra ecuatoriana, se obtuvo un promedio de 6,80 $\pm 1,11 \mathrm{~g}$, siendo valores superiores a los reportados en gallinas criollas alimentadas a base de maíz (Juárez-Caratachea et al. 2010) reportando $4,5 \pm 0,3 \mathrm{~g}$, en el peso de la cás- 
Tabla III. Análisis de la hipótesis con chi cuadrado (Analysis of the hypothesis with chi square).

\begin{tabular}{lllll} 
& \multicolumn{3}{c}{ Chi Cuadrado } \\
Chi cuad. Calculado & 5,6 & Ho & No existe diferencia \\
Chi cuad. Tabulado & 11,07 & Hi & Si existe diferencia \\
GL: 5 & & & \\
P: 0.05 & & & \\
\hline
\end{tabular}

cara de las gallinas criollas esto se atribuye a la alimentación con balanceado, cubriendo el requerimiento de calcio y fósforo, principales componentes de la cáscara, para el grosor de la cáscara de las gallinas criollas de la Sierra ecuatoriana, se obtuvo un promedio de 0,32 $\pm 0,02 \mathrm{~mm}$, superando estos resultados a los reportados por (Juárez-Caratachea et al. 2010), con 0,28 \pm $0,09 \mathrm{~mm}$, esta diferencia se debe a que el sistema de producción avícola de traspatio se basa en una dieta de grano (maíz), lo cual suele provocar limitaciones de calcio y fósforo, minerales estructurales de la cáscara que puede repercutir sobre el grosor de la misma, también menciona que el estado nutricional, la edad de la gallina, ritmo de postura, tamaño del huevo, estado de salud y manejo, son otros factores de influencia en el grosor de la cáscara, en otro estudio con huevos de color azul, a la vez (Juárez-Caratachea et al. 2010), reporta efectos para gallinas de emplume normal, un grosor de cáscara de 0,35 $\pm 0,07 \mathrm{~g}$, mayor al reportado en la presente investigación, los principales factores que afectan a la calidad de la cáscara son; genética, edad de los animales, nutrición, factores de estrés y el sistema de producción.

La variable ancho del huevo en lo que, respecto a la variable largo del huevo en las gallinas criollas de la Sierra ecuatoriana, se obtuvo un promedio de 55,40 \pm 2,60 mm siendo superior a los reportados por (JuárezCaratachea et al. 2010) en los dos casos.

\section{CONCLUSION}

Las gallinas criollas captadas en las diferentes provincias de la región sierra de Ecuador presentaron diferentes pesos iniciales, aun cuando son de edades similares lo que ha influenciado en su ganancia de peso y su peso final, dado los diferentes medio ambientes donde se desarrollaron, lo que ha con llevado a obtener huevos de diferentes pesos y tamaños, así como también el estrés por adaptación que sufrieron al ser trasladadas al lugar de investigación; cabe indicar que la característica de rusticidad, es de interés, representando gran valor para la conservación de los recursos genéticos, las aves criollas han demostrado poseer una buena capacidad de adaptación al medio a lo largo del tiempo, que aseguran rendimientos productivos.

\section{AGRADECIMIENTOS}

A la Escuela Superior Politécnica de Chimborazo, Carrera de Zootecnia, Estación Experimental Tunshi; así como también al grupo AGR18 Departamento de Genética de la Universidad de Córdoba. Y de manera especial a todos los productores de traspatio del Ecua- dor, que nos permitieron realizar varias visitas a sus explotaciones y adquirir sus aves.

\section{BIBLIOGRAFÍA}

Alderson, GLH 2018, 'Conservation of breeds and maintenance of biodiversity: justification and methodology for the conservation of Animal Genetic Resources', Archivos de Zootecnia, vol. 67, no. 258.

Andrade-Yucailla V., A-CC, Ramírez A., Viamonte M.I., Sánchez J., \& Toalombo-Vargas P., Á-PGR, Vargas-Burgos J.C. 2018, 'Caracterización morfométrica y faneróptica de la gallina criolla (Gallus domesticus), en traspatios familiares del cantón Santa Clara, Pastaza', AICA, vol. 12, pp. 1-8.

FAO 2015, 'El huevo en cifras', ww.fawo.org. Recuperado de http:// www.fao.org/assets/infographics/FAO-Infographic-egg-facts-es.pdf 22/04/2015 Roma. Italia

Formoso-Rafferty, N, García-García, RM, Rodríguez, M, Alonso, A, Masdeu, M, Millán, P, Arias-Álvarez, M, Lorenzo, PL \& Rebollar, PG 2016, 'Caracterización de parámetros reproductivos y endocrinos en una población de conejas ibicencas', Archivos de Zootecnia, vol. 65 , no. 252, pp. 525-34.

Juárez-Caratachea, A, Gutiérrez-Vázquez, E, Segura-Correa, J \& Santos-Ricalde, R 2010, 'Calidad del huevo de gallinas criollas criadas en traspatio en Michoacán, México', Tropical and subtropical agroecosystems, vol. 12, no. 1, pp. 109-15.

Lázaro, GC, Hernández, ZIS, Vargas, LS, Martínez, LA \& Pérez, AR 2012, 'Uso de caracteres morfométricos en la clasificación de gallinas locales', Actas Iberoamericanas de Conservación Animal, vol. 2, no. 1, pp. 109-14.

Luka, SA \& Ndams, IS 2007, 'Short communication report: Gastrointestinal parasites of domestic chicken Gallus-gallus domesticus linnaeus 1758 in Samaru, Zaria Nigeria', Science World Journal, vol. 2, no. 1.

Martínez Correal, G 2010, 'Plan nacional de acción para la conservación, mejoramiento y utilización sostenible de los recursos genéticos animales de Colombia: informe final/Recurso electrónico'.

Monterrubio, RAD 2000, 'Lombriz roja (Eisenia spp), alternativa sustentable en la alimentación de gallinas criollas'.

Moula, N, Philippe, F-X, Moussiaux, NA, Leroy, P \& Michaux, C 2014, 'Estimation of inbreeding rates and extinction risk of forty one belgian chicken breeds in 2005 and 2010', Archivos de Zootecnia, vol. 63, no. 242, pp. 389-92.

Rodríguez Buenfil, JC, Allaway, CE, Wassink, GJ, Segura Correa, JC \& Rivera Ortega, T 1996, 'Estudio de la avicultura de traspatio en el municipio de Dzununcán, Yucatán', Vet. Méx, vol. 27, no. 3, pp. 215-9.

Rodríguez García, R 2007, Agricultura tropical con enfoque humano y visión sistémica.

Salas, MPJ, Sánchez, MR, Rodríguez, JCC, Aparicio, YV \& Correa, JS 2009, 'Indicadores productivos de gallinas criollas en un sistema de producción avicola alternativo en Oaxaca, Mexico'.

Toscano, RL 2002, 'Efecto de tres dietas sobre los parámetros productivos y reproductivos en gallinas criollas'.

Vignon, CL 1997, 'Variables de selección en huevos criollos que influyen en incubabilidad, calidad y producción de pollo'.

Vignon, CL \& Santiago, DH 1996, 'Características reproductivas de gallinas criollas en condiciones controladas y de traspatio en el Valle de Zaachila, Oax'.

Villacís Rivas, G, Escudero Sánchez, G, Cueva Castillo, F \& Luzuriaga Neira, A 2016, 'Características morfométricas de las gallinas criollas de comunidades rurales del sur del Ecuador', Revista de Investigaciones Veterinarias del Perú, vol. 27, no. 2, pp. 218-24. 